\title{
Synthesis of Polynuclear Heterocyclic Compounds Derived from Thieno[2,3-d]pyrimidine Derivatives
}

\author{
A.B.A. El-Gazzar and N.A. Hassan* \\ Photochemistry Department, National Research Center, Cairo, Egypt \\ Fax: 002-02-3370931, E-mail: nasserabdelhamid@hotmail.com
}

Received: 2 August 1999; revised form 6 June 2000/Accepted: 13 June 2000 /Published: 25 June 2000

\begin{abstract}
Reaction of 2-hydrazino-3-methyl-3,4-dihydrothieno[2,3- $d$ ]pyrimidin-4-one derivatives $\mathbf{2 a}, \mathbf{b}$ with aliphatic acids afforded the thienotriazolopyrimidinone derivatives 3a-d, with nitrous acid yielded tetrazolothienopyrimidinone derivatives $\mathbf{4 a}, \mathbf{b}$ and with carbon disulphide furnished 3-mercaptothienotriazolopyrimidinone derivatives 5a,b. Also, 2a,b reacted with aldehydes to afford the arylhydrazones $\mathbf{6 a - f}$ which cyclized into thienotriazolopyrimidinone derivatives 7a-f. Furthermore, 2a,b condensed with ethyl acetoacetate and ethyl cyanoacetate to afford 2-(1-pyrazolyl) derivatives 9a,b and 10a,b, respectively. On the other hand, 2-hydrazino derivatives $\mathbf{2 a}, \mathbf{b}$ condensed with $\alpha$-halo-ketones to yield thienpyrimidotriazinone derivatives 11a,b and with $\beta$-diketones, to form 2-(1-pyrazolyl) derivatives 12a-f.
\end{abstract}

Keywords: Pyrimidines, $\alpha$-haloketones, $\beta$-diketones, $\beta$-ketoesters, aliphatic acids, NMR spectra.

\section{Introduction}

The biological [1-5], bactericidal [6], and medicinal [7,8] activities of thieno[2,3- $d$ ]-pyrimidine derivatives have stimulated considerable research in this field [9-12]. In continuation of our work on the synthesis of fused pyrimidine derivatives [13], we report here the utility of 3-methyl-2-methylthio-3,4dihydrotheino[2,3- $d$ ]pyrimidine-4-one derivatives 1a,b for the synthesis of azolothienopyrimid-ines, thienopyrimido-as-triazines, pyrazolylthienopyrimidines and tetrazolothienopyrimidines.

(C) 2000 by MDPI (http://www.mdpi.org). Reproduction is permitted for noncommercial purposes. 


\section{Results and Discussion}

As shown in Scheme 1, heating under reflux a solution of 3-methyl-2-methylthio-3,4-dihydrothieno[2,3- $d$ ] pyrimidin-4-one derivatives 1a,b in ethanol with excess hydrazine hydrate yielded the corresponding 2-hydrazino-3-methyl-3,4-dihydrothieno[2,3-d]pyrimidin-4-one derivatives 2a,b [2]. The latter compounds are considered as key intermediates for the synthesis of some new azolothienopyrimidines, thienopyrimido-as-triazines as well as the synthesis of some pyrazolylthienopyrimidine derivatives.<smiles>[R]c1sc2c(c1[R])c(=O)n(C)c1nnc([R])n21</smiles>

3a, $\mathrm{R}_{1}=\mathrm{R}_{2}=\mathrm{CH}_{3}, \quad \mathrm{R}=\mathrm{H}$

$3 \mathrm{~b}, \mathrm{R}_{1}+\mathrm{R}_{2}=\left(\mathrm{CH}_{2}\right)_{4}, \mathrm{R}=\mathrm{H}$

3c, $\mathrm{R}_{1}=\mathrm{R}_{2}=\mathrm{CH}_{3}, \quad \mathrm{R}=\mathrm{CH}_{3}$ 3d, $\mathrm{R}_{1}+\mathrm{R}_{2}=\left(\mathrm{CH}_{2}\right)_{4}, \mathrm{R}=\mathrm{CH}_{3}$

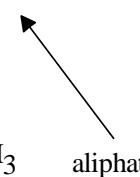

aliphatic acid<smiles>[R]c1sc2nc(NC=[W])n(C)c(=O)c2c1[R]</smiles>

6a-f

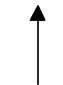

Ar.CHO

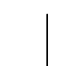<smiles>[R]c1sc2c(c1[2H])c(=O)n(C)c1nnc([Al])n21</smiles>

6,7a, $\mathrm{R}_{1}=\mathrm{R}_{2}=\mathrm{CH}_{3}, \quad \mathrm{Ar}=\mathrm{C}_{6} \mathrm{H}_{5}$ 6,7b, $\mathrm{R}_{1}=\mathrm{R}_{2}=\mathrm{CH}_{3}, \quad \mathrm{Ar}=\mathrm{C}_{6} \mathrm{H}_{4} \cdot \mathrm{Cl}-p$ 6,7c, $\mathrm{R}_{1}=\mathrm{R}_{2}=\mathrm{CH}_{3}, \quad \mathrm{Ar}=\mathrm{C}_{6} \mathrm{H}_{4} \cdot \mathrm{OCH}_{3}-p$ 6,7d, $\mathrm{R}_{1}+\mathrm{R}_{2}=\left(\mathrm{CH}_{2}\right)_{4}, \mathrm{Ar}=\mathrm{C}_{6} \mathrm{H}_{5}$ 6,7e, $\mathrm{R}_{1}+\mathrm{R}_{2}=\left(\mathrm{CH}_{2}\right)_{4}, \mathrm{Ar}=\mathrm{C}_{6} \mathrm{H}_{4} \cdot \mathrm{Cl}-p$ 6,7f, $\mathrm{R}_{1}+\mathrm{R}_{2}=\left(\mathrm{CH}_{2}\right)_{4}, \mathrm{Ar}=\mathrm{C}_{6} \mathrm{H}_{4} \cdot \mathrm{OCH}_{3}-p$<smiles>[R]c1sc2nc(NC(=O)CCC(=O)OCC)n(C)c(=O)c2c1[R]</smiles><smiles>[R]c1sc2nc(N3N=C(C)CC3=O)n(C)c(=O)c2c1[R]</smiles>

8a,9a, $\mathrm{R}_{1}=\mathrm{R}_{2}=\mathrm{CH}_{3}$ 8a,9b, $\mathrm{R}_{1}+\mathrm{R}_{2}=\left(\mathrm{CH}_{2}\right)_{4}$<smiles>[R]c1sc2nc(N)n(C)c(=O)c2c1[R]C</smiles>

1a, $2 \mathrm{a}, \mathrm{R}_{1}=\mathrm{R}_{2}=\mathrm{CH}_{3}$ $\mathbf{1 a}, \mathbf{2 b}, \mathrm{R}_{1}+\mathrm{R}_{2}=\left(\mathrm{CH}_{2}\right)_{4}$<smiles></smiles>

$4 \mathrm{a}, 5 \mathrm{a}, \mathrm{R}_{1}=\mathrm{R}_{2}=\mathrm{CH}_{3}$ $4 \mathrm{a}, 5 \mathbf{b}, \mathrm{R}_{1}+\mathrm{R}_{2}=\left(\mathrm{CH}_{2}\right)_{4}$<smiles>[R]c1sc2c(c1[R])c(=O)n(C)c1nnnn21</smiles><smiles>[R]c1sc2nc(N)n(C)c(=O)c2c1[R]</smiles><smiles>[13CH][13CH3]</smiles>

a, $\mathbf{R}_{1}=\mathbf{R}_{2}=\mathbf{C H}_{3}, \mathbf{b}, \mathbf{R}_{1}+\mathbf{R}_{2}=\left(\mathbf{C H}_{2}\right)_{4}$<smiles>[R]c1sc2nc(N)n(C)c(=O)c2c1[R]</smiles>

4 a,b

Scheme 1. 
Heating under reflux compounds $\mathbf{2 a}, \mathbf{b}$ with aliphatic acids, namely formic or acetic acid, resulted in the formation of the corresponding 4,5-dihydrothieno [3,2-e][1,2,4]triazolo[3,4-a]-pyrimidin-5-one derivatives 3a-d. Besides the correct values of elemental analyses, the IR, ${ }^{1} \mathrm{H}-\mathrm{NMR}$ and ${ }^{13} \mathrm{C}-\mathrm{NMR}$ spectra of 3a-d are in agreement with the assigned structures, (c.f. Experimental and Scheme 1).

Treatment of compounds $\mathbf{2 a}, \mathbf{b}$ with nitrous acid at $0^{\circ} \mathrm{C}$, led to the formation of the corresponding tetrazolo[1,5-b]thieno[2,3- $d$ ]pyrimidin-5-one derivatives $\mathbf{4 a , b}$ having a new ring system, which could be found in equilibrium with the 2-azido-3-methyl-3,4-dihydrothieno[2,3- $d$ ]-pyrimidin-4-one tautomer [14]. The IR spectrum of $\mathbf{4 a}$, as an example, displayed absorption bands at $1713 \mathrm{~cm}^{-1}$ (corresponding to $-\mathrm{C}=\mathrm{O}$ ) and at $2240 \mathrm{~cm}^{-1}$ (characteristic of the absorption of the azido group). Also, the ${ }^{1} \mathrm{H}-\mathrm{NMR}$ and ${ }^{13} \mathrm{C}$-NMR confirmed the assigned structures $\mathbf{4 a}, \mathbf{b}$ (c.f. Experimental and Scheme 1).

Moreover, the 2-aminopyrimidine derivatives are reported in the literature to have varied biological activities, acting as anticancer, antibacterial and antimalaria agents [14]. Therefore compounds $\mathbf{4 a , b}$ were reduced to the corresponding 2-amino-3-methyl-3,4-dihydrothieno[2,3-d]-pyrimidin-4-ones 4-a,b by zinc dust and acetic acid. The IR spectrum of $\mathbf{4} \mathbf{a}$ as an example displayed absorption band at 3280 $\mathrm{cm}^{-1}$ corresponding to $-\mathrm{NH}_{2}$, besides compatible ${ }^{1} \mathrm{H}-\mathrm{NMR},{ }^{13} \mathrm{C}-\mathrm{NMR}$ and microanalytical data, (c.f. Experimental and Scheme 1).

Also, compounds $\mathbf{2 a , b}$ reacted with carbon disulfide in ethanolic sodium hydroxide to afford the mercaptothieno[3,2-e][1,2,4]triazolo[3,4-a]pyrimidin-5-one derivatives $\mathbf{5 a}, \mathbf{b}$ respectively, as indicated by the analytical data (c.f. Experimental and Scheme 1). The interaction of $\mathbf{2 a}, \mathbf{b}$ with a suitable aldehyde in boiling dioxane in the presence of catalytic amounts of piperidine afforded the arylhydrazones $6 \mathbf{a}-\mathbf{f}$ which could be cyclized to the aryl-4,5-dihydrothieno-[3,2-e][1,2,4]triazolo[3,4-e]pyrimidin-5-one derivatives 7a-f when they were treated with an excess of bromine in acetic acid in the presence of anhydrous sodium acetate. Structures $\mathbf{6 a - f}$ and 7a-f were confirmed by their correct microanalyses and compatible spectroscopic data, (c.f. Experimental and Scheme 1).

Boiling an ethanolic solution of $\mathbf{2 a}, \mathbf{b}$ with ethyl acetoacetate for $2 \mathrm{hr}$ afforded the hydrazone derivatives 8a,b which could be cyclized by heating in ethanolic sodium ethoxide solution to give compounds 9a,b respectively. Similarly, the 2-(pyrazolyl) derivatives $\mathbf{1 0 a}, \mathbf{b}$ were isolated in good yields upon treatment of $\mathbf{2 a}, \mathbf{b}$ with ethyl cyanoacetate. All spectroscopic data is in agreement with the assigned structures 9a,b and 10a,b (c.f. Experimental and Schemes 1 and 2).

Heating compounds $\mathbf{2 a}, \mathbf{b}$ with $\alpha$-haloketones (chloroacetone or phenacyl bromide) in dry xylene, yielded the corresponding compounds 11a,b respectively, which have a new ring system. In addition, compounds 2a,b condensed with each of pentane-2,4-dione, 3-chloropentane-2,4-dione or 1,1,1trifluoropentane-2,4-dione in absolute ethanol, yielding the corresponding compounds 12a-f. Besides the correct values of their elemental analyses, the analytical data is in agreement with all the assigned structures, (c.f. Experimental and Scheme 2). 


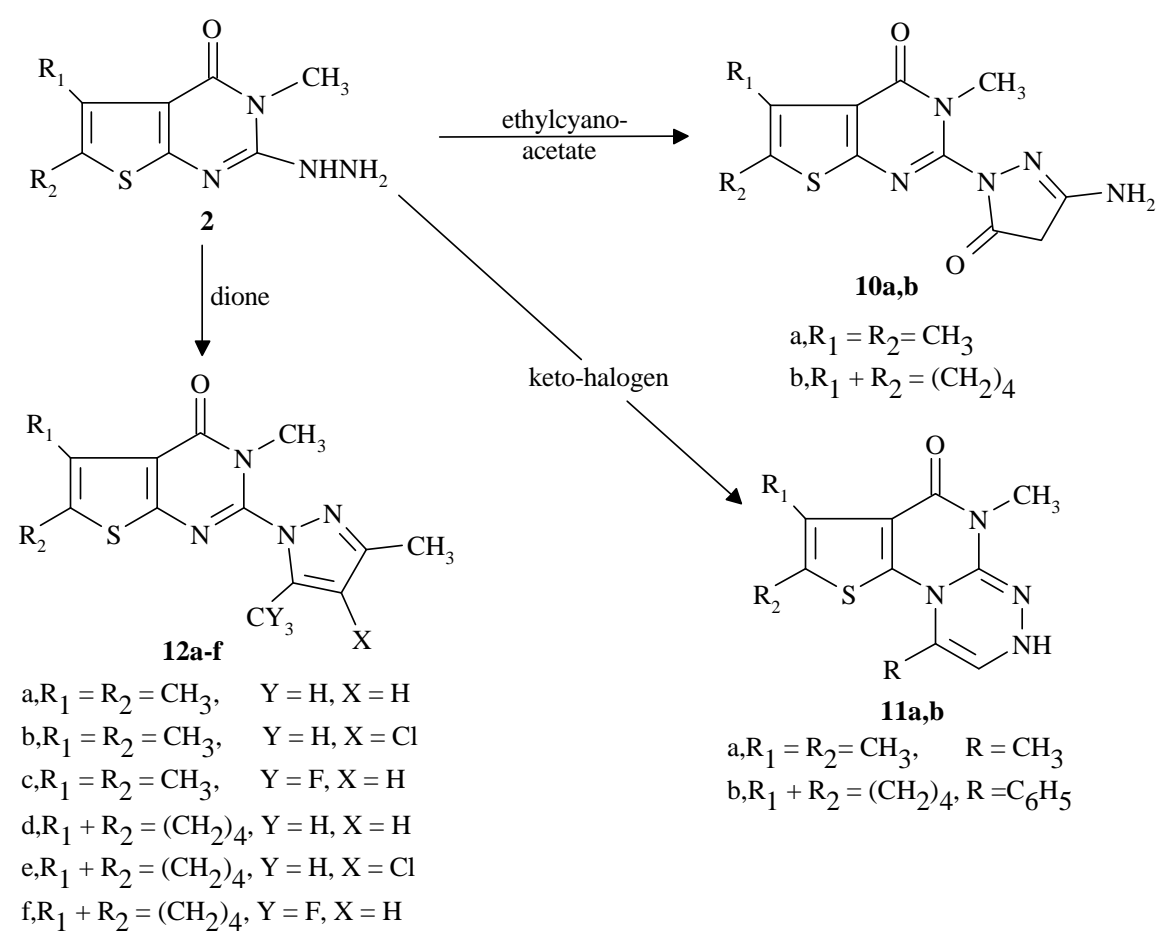

Scheme 2.

\section{Experimental}

\section{General}

All melting points are uncorrected. The ${ }^{1} \mathrm{H}-\mathrm{NMR}$ and ${ }^{13} \mathrm{C}-\mathrm{NMR}$ spectra were recorded on Bruker (WM-250 MHz), Bruker (AC-250 MHz) spectrometers (Faculty of Chemistry, Konstanz University, Germany), and a Varian ${ }^{1} \mathrm{H}$ Gemini 200 spectrometer (National Research Center, Egypt) and chemical shifts were expressed as $\delta$ values against $\mathrm{SiMe}_{4}$ used as internal standard. IR spectra were recorded as potassium bromide pellets on a Perkin-Elmer 1430 spectrometer, (National Research Center and Department of Chemistry, Cairo University) and Perkin-Elmer 1320 and 299 spectrometers (Faculty of Chemistry, Konstanz University, Germany). Mass spectra were recorded on a Shimadzu (Japan) GCMS-QP 1000 EX gas chromatography-mass spectrometer system. Microanalytical data were obtained by the Microanalytical Center at Konstanz University (Germany), Cairo University and National Research Center (Egypt).

General procedure for the preparation of $\mathbf{3 a}, \mathbf{b}$

A mixture of compound $\mathbf{2 a}$ or $\mathbf{2 b}$ [2] (10 mmol), formic acid (10 mL) and catalytic amount of concentrated hydrochloric acid was heated under reflux for $5 \mathrm{~h}$. The reaction mixture was allowed to cool to room temperature and poured into water $(100 \mathrm{~mL})$. The formed solid was collected by filtration, washed with ethanol, dried and crystallized from the proper solvent. 


\section{4,6,7-Trimethyl-4,5-dihydrothieno[3,2-e][1,2,4]triazolo[3,4-a]pyrimidin-5-one (3a)}

From 2a, crystallized from dioxane $(50 \mathrm{~mL})$ in $73 \%$ yield, m.p. $267-9^{\circ} \mathrm{C}$; IR $(\mathrm{KBr}) \mathrm{cm}^{-1}: 2980(\mathrm{CH}$ alkyl), $1689(\mathrm{C}=\mathrm{O}), 1576(\mathrm{C}=\mathrm{N}), 1519(\mathrm{C}=\mathrm{C}) ;{ }^{1} \mathrm{H}-\mathrm{NMR}$ (DMSO- $\left.d_{6}\right) \mathrm{ppm}$ : $\delta 2.55\left(\mathrm{~s}, 6 \mathrm{H}, 2 \mathrm{CH}_{3}\right), \delta 3.86$ $\left(\mathrm{s}, 3 \mathrm{H}, \mathrm{N}-\mathrm{CH}_{3}\right), \delta 8.58\left(\mathrm{~s}, 1 \mathrm{H}\right.$, methylenic proton); ${ }^{13} \mathrm{C}-\mathrm{NMR}$ (DMSO- $\left.d_{6}\right): 13.22,13.29\left(2 \mathrm{CH}_{3}\right), 31.19$ $\left(\mathrm{N}-\mathrm{CH}_{3}\right)$ 122.85-148.50 (6C, sp ${ }^{2}$ carbon atoms), $157.55(\mathrm{C}=\mathrm{O})$. Analyses: $\mathrm{C}_{10} \mathrm{H}_{10} \mathrm{~N}_{4} \mathrm{OS}(234.3)$. Required: C, 51.27; H, 4.31; N, 23.92; Found: C, 51.19; H, 4.28; N, 23.64.

\section{4-Methyl-6,7,8,9-tetrahydrobenzo-4,5-dihydrothieno[3,2-e][1,2,4]triazolo[3,4-a]pyrimidin-5-one (3b)}

From $2 \mathbf{b}$, crystalized from dioxane $(40 \mathrm{ml})$ in $76 \%$ yield, m.p. $243-45^{\circ} \mathrm{C}$; IR $(\mathrm{KBr}) \mathrm{cm}^{-1}: 2983(\mathrm{CH}$ alkyl), $1669(\mathrm{C}=\mathrm{O}), 1581(\mathrm{C}=\mathrm{N}), 1509(\mathrm{C}=\mathrm{C}) ;{ }^{1} \mathrm{H}-\mathrm{NMR}\left(\mathrm{DMSO}-d_{6}\right) \mathrm{ppm}: \delta 1.92\left(\mathrm{~m}, 4 \mathrm{H}, 2 \mathrm{CH}_{2}\right), \delta$ $2.78\left(\mathrm{t}, 2 \mathrm{H}, \mathrm{CH}_{2}\right), \delta 3.00\left(\mathrm{t}, 2 \mathrm{H}, \mathrm{CH}_{2}\right), \delta 3.73\left(\mathrm{~s}, 3 \mathrm{H}, \mathrm{N}-\mathrm{CH}_{3}\right), \delta 8.40$ (s, $1 \mathrm{H}$, methylenic proton); ${ }^{13} \mathrm{C}-$ NMR (DMSO-d $\left.)_{6}\right)$ 21.83-25.53 $\left(4 \mathrm{CH}_{2}\right), 29.19\left(\mathrm{~N}^{-\mathrm{CH}_{3}}\right)$ 118.34-148.96 (6C, $\mathrm{sp}^{2}$ carbon atoms), 156.30 $(\mathrm{C}=\mathrm{O})$. Analyses: $\mathrm{C}_{12} \mathrm{H}_{12} \mathrm{~N}_{4} \mathrm{OS}$ (260.3). Required: C, 55.37; H, 4.66; N, 21.53; Found: C, 55.19; H, $4.52 ; \mathrm{N}, 20.98$.

General procedure for the preparation of $\mathbf{3 c}, \mathbf{d}$

A mixture of $\mathbf{2 a}$ or $\mathbf{2 b}(10 \mathrm{mmol})$ and glacial acetic acid $(30 \mathrm{~mL})$ was stirred under reflux for $18 \mathrm{hr}$. The reaction mixture was allowed to cool to room temperature and poured into water $(100 \mathrm{~mL})$. The solid thus formed was collected by filtration, washed with ethanol $(20 \mathrm{~mL})$, dried, and crystallized from acetic acid.

\section{1,4,6,7-Tetramethyl-4,5-dihydrothieno[3,2-e][1,2,4]triazolo[3,4-a]pyrimidin-5-one (3c)}

From 2a in 69\% yield, m.p. $226-29^{\circ} \mathrm{C}$; IR $(\mathrm{KBr}) \mathrm{cm}^{-1}: 2980(\mathrm{CH}$ alkyl), $1650(\mathrm{C}=\mathrm{O}), 1580(\mathrm{C}=\mathrm{N})$, $1500(\mathrm{C}=\mathrm{C}) ;{ }^{1} \mathrm{H}-\mathrm{NMR}\left(\mathrm{TFA}: \mathrm{CDCl}_{3} / 1: 1\right) \mathrm{ppm}: \delta 2.55\left(\mathrm{~s}, 6 \mathrm{H}, 2 \mathrm{CH}_{3}\right), \delta 3.08\left(\mathrm{~s}, 3 \mathrm{H}, \mathrm{CH}_{3}\right), \delta 3.76(\mathrm{~s}, 3 \mathrm{H}$,

$\left.\mathrm{N}-\mathrm{CH}_{3}\right) ;{ }^{13} \mathrm{C}-\mathrm{NMR}: 11.22,12.97,13.05\left(3 \mathrm{CH}_{3}\right), 30.50\left(\mathrm{~N}^{\left.-\mathrm{CH}_{3}\right)} 122.16-148.55\right.$ (6C sp ${ }^{2}$ carbon atoms), $157.28(\mathrm{C}=\mathrm{O})$. Analyses: $\mathrm{C}_{11} \mathrm{H}_{12} \mathrm{~N}_{4} \mathrm{OS}$ (248.3). Required: C, 53.21; H, 4.88; N, 22.57; Found: C, 53.15; H, 4.49; N, 22.2.

1,4-Dimethyl-6,7,8,9-tetrahydrobenzo-4,5-dihydrothieno[3,2-e][1,2,4]triazolo[3,4-a]pyrimidin-5-one (3d)

From $2 b$ in $73 \%$ yield, m.p. $238-41^{\circ} \mathrm{C}$; IR $(\mathrm{KBr}) \mathrm{cm}^{-1}$ : $2956(\mathrm{CH}$ alkyl), $1687(\mathrm{C}=\mathrm{O}), 1562(\mathrm{C}=\mathrm{N})$, $1522(\mathrm{C}=\mathrm{C}) ;{ }^{1} \mathrm{H}-\mathrm{NMR}\left(\mathrm{TFA}: \mathrm{CDCl}_{3} / 1: 1\right) \mathrm{ppm}: \delta 1.96\left(\mathrm{~m}, 4 \mathrm{H}, 2 \mathrm{CH}_{2}\right), \delta 2.22\left(\mathrm{~s}, 3 \mathrm{H}, \mathrm{CH}_{3}\right), \delta 2.93(\mathrm{t}$, $2 \mathrm{H}, \mathrm{CH}_{2}$ ), $\delta 3.09$ (t, 2H, $\mathrm{CH}_{2}$ ), $\delta 3.77$ (s, 3H, N-CH ); Analyses: $\mathrm{C}_{13} \mathrm{H}_{14} \mathrm{~N}_{4} \mathrm{OS}$ (274.4). Required: C, 56.92; H, 5.15; N, 20.42; Found: C, 56.81; H, 5.21; N, 20.13. 
General procedure for the preparation of $\mathbf{4 a}, \mathbf{b}$

A solution of sodium nitrite $(1.04 \mathrm{~g}, 15 \mathrm{mmol})$ in the least amount of water was added dropwise to an an ice-cold solution of compound $\mathbf{2 a}$ or $\mathbf{2 b}(10 \mathrm{mmol})$ in acetic acid $(10 \mathrm{~mL}) \mathrm{kept}$ an ice bath at $-5^{\circ} \mathrm{C}$. The reaction mixture was allowed to stand overnight at room temperature, then it was poured into water $(100 \mathrm{~mL})$. The solid so-precipitated was filtered off and crystallized from benzene.

\section{4,6,7-Trimethyl-4,5-dihydrothieno[3,2-e][1,2,3,4]tetrazolo[1,5-a]pyrimidin-5-one (4a)}

From $2 \mathbf{a}$ in $53 \%$ yield, m.p. $183-85^{\circ} \mathrm{C}$ (dec.); IR (KBr) cm ${ }^{-1}: 2954(\mathrm{CH}$ alkyl), $1713(\mathrm{C}=\mathrm{O}), 1629$ $(\mathrm{N}=\mathrm{N}), 1582(\mathrm{C}=\mathrm{N}), 1506(\mathrm{C}=\mathrm{C}) ;{ }^{1} \mathrm{H}-\mathrm{NMR}\left(\mathrm{DMSO}-d_{6}\right) \mathrm{ppm}: \delta 2.47\left(\mathrm{~s}, 6 \mathrm{H}, 2 \mathrm{CH}_{3}\right), \delta 3.88(\mathrm{~s}, 3 \mathrm{H}, \mathrm{N}-$ $\left.\mathrm{CH}_{3}\right) ;{ }^{13} \mathrm{C}-\mathrm{NMR}: 11.22,12.97\left(2 \mathrm{CH}_{3}\right), 30.53\left(\mathrm{~N}_{-} \mathrm{CH}_{3}\right)$ 118.22-148.55 (5C, $\mathrm{sp}^{2}$ carbon atoms), 157.28 $(\mathrm{C}=\mathrm{O})$. Analyses: $\mathrm{C}_{9} \mathrm{H}_{9} \mathrm{~N}_{5} \mathrm{OS}$ (235.3). Required: C, 45.94; H, 3.86; N, 29.77; Found: C, 45.81; H, 3.65; $\mathrm{N}, 30.05$.

4-Methyl-6,7,8,9-tetrahydrobenzo-4,5-dihydrothieno[3,2-e][1,2,3,4]tetrazolo[1,5-a]pyrimidin-5-one (4b)

From $2 b$ in 61\% yield, m.p. 210-12 ${ }^{\circ} \mathrm{C}$ (dec.); IR (KBr) cm ${ }^{-1}: 2943(\mathrm{CH}$ alkyl), $1698(\mathrm{C}=\mathrm{O}), 1609$ $(\mathrm{N}=\mathrm{N}), 1567(\mathrm{C}=\mathrm{N}), 1520(\mathrm{C}=\mathrm{C}) ;{ }^{1} \mathrm{H}-\mathrm{NMR}\left(\mathrm{DMSO}-d_{6}\right) \mathrm{ppm}: \delta 1.97\left(\mathrm{~m}, 4 \mathrm{H}, 2 \mathrm{CH}_{2}\right), \delta 2.95(\mathrm{t}, 2 \mathrm{H}$, $\mathrm{CH}_{2}$ ), $\delta 3.19$ (t, 2H, $\mathrm{CH}_{2}$ ), $\delta 3.82$ (s, 3H, N-CH ); Analyses: $\mathrm{C}_{11} \mathrm{H}_{11} \mathrm{~N}_{5} \mathrm{OS}$ (261.3). Required: C, 50.56; H, 4.25; N, 26.81; Found: C, 50.32; H, 4.11; N, 27.01.

General procedure for the preparation of $\mathbf{4} \mathbf{a}, \mathbf{b}$

Activated zinc dust $(5.00 \mathrm{~g})$ was added protionwise to a well stirred solution the appropriate tetrazolo-thienopyrimidine $\mathbf{4 a}$ or $\mathbf{4 b}(10 \mathrm{mmol})$ in glacial acetic acid $(30 \mathrm{~mL})$ at room temperature over a period of 30 minutes. Stirring was continued for additional $3 \mathrm{hr}$. Then the reaction mixture was heated on a water bath $\left(80-90^{\circ} \mathrm{C}\right)$ for $3 \mathrm{hr}$. The progress of reduction was monitored by TLC. After allowing the reaction mixture to cool to room temperature, it was poured into cold water $(100 \mathrm{~mL})$. The insoluble solid which separated was filtered, washed with water and dried. The crude solid was extracted with hot benzene and the solid obtained after removal of benzene was crystallized from acetic acid.

\section{2-Amino-3,5,6-trimethyl-3,4-dihydrothieno[2,3-d]pyrimidin-4-one (4)a)}

From 4a, in $49 \%$ yield, m.p. $321-24^{\circ} \mathrm{C}$; IR (KBr) cm ${ }^{-1}$ : 3280 (brs, $\left.\mathrm{NH}_{2}\right), 2923(\mathrm{CH}$ alkyl), 1686 $(\mathrm{C}=\mathrm{O}), 1593(\mathrm{C}=\mathrm{N}), 1557(\mathrm{C}=\mathrm{C}) ;{ }^{1} \mathrm{H}-\mathrm{NMR}\left(\mathrm{DMSO}-d_{6}\right) \mathrm{ppm}: \delta 2.07\left(\mathrm{~s}, 3 \mathrm{H}, \mathrm{CH}_{3}\right), \delta 2.31\left(\mathrm{~s}, 3 \mathrm{H}, \mathrm{CH}_{3}\right)$, $\delta 3.88\left(\mathrm{~s}, 3 \mathrm{H}, \mathrm{N}-\mathrm{CH}_{3}\right), \delta 3.25$ (brs, $2 \mathrm{H}, \mathrm{NH}_{2}, \mathrm{D}_{2} \mathrm{O}$ exchangeable); ${ }^{13} \mathrm{C}-\mathrm{NMR}: 11.24,12.96\left(2 \mathrm{CH}_{3}\right)$, $30.54\left(\mathrm{~N}_{-} \mathrm{CH}_{3}\right), 118.3-148.61\left(5 \mathrm{C} \mathrm{sp}{ }^{2}\right.$ carbon atoms), $160.12(\mathrm{C}=\mathrm{O})$. Analyses: $\mathrm{C}_{9} \mathrm{H}_{11} \mathrm{~N}_{3} \mathrm{OS}(209.3)$. Required: C, 51.64; H, 5.31; N, 20.08; Found: C, 51.49; H, 5.17; N, 19.79. 


\section{2-Amino-3-methyl-5,6,7,8-tetrahydrobenzo-3,4-dihydrothieno[2,3-d]pyrimidin-4-one (4)b)}

From 4b, in 51\% yield, m.p. 298-301 ${ }^{\circ} \mathrm{C}$; $\mathrm{R}(\mathrm{KBr}) \mathrm{cm}^{-1}$ : 3245 (brs, $\left.\mathrm{NH}_{2}\right), 2953(\mathrm{CH}$ alkyl), 1694 $(\mathrm{C}=\mathrm{O}), 1560(\mathrm{C}=\mathrm{N}), 1541(\mathrm{C}=\mathrm{C}) ;{ }^{1} \mathrm{H}-\mathrm{NMR}\left(\mathrm{DMSO}-d_{6}\right) \mathrm{ppm}: \delta 1.94\left(\mathrm{~m}, 4 \mathrm{H}, 2 \mathrm{CH}_{2}\right), \delta 2.91(\mathrm{t}, 2 \mathrm{H}$, $\mathrm{CH}_{2}$ ), $\delta 3.17$ (t, 2H, $\mathrm{CH}_{2}$ ), $\delta 3.87$ (s, 3H, N-CH ), 10.50 (brs, $2 \mathrm{H}, \mathrm{NH}_{2}, \mathrm{D}_{2} \mathrm{O}$ exchangeable). Analyses: $\mathrm{C}_{11} \mathrm{H}_{13} \mathrm{~N}_{3} \mathrm{OS}$ (235.3). Required: C, 56.15; H, 5.58; N, 17.86; Found: C, 55.98; H, 5.39; N, 17.91.

General procedure for the preparation of $\mathbf{5 a}, \mathbf{b}$

To a warmed ethanolic sodium hydroxide solution prepared by dissolving of sodium hydroxide $(0.40 \mathrm{~g}, 10 \mathrm{mmol})$ in ethanol $(50 \mathrm{~mL})$ was added $(10 \mathrm{mmol})$ of compound $(\mathbf{2 a}, \mathbf{b})$ and excess carbon disulphide $(10 \mathrm{~mL})$. The mixture was refluxed in a water bath at $80^{\circ} \mathrm{C}$ for $10 \mathrm{hr}$, then allowed to cool to room temperature, poured into water $(100 \mathrm{~mL})$, neutralized by dilute acetic acid and the precipitate formed was filtered off and dried. The product was crystallized from benzene.

\section{4,6,7-Trimethyl-1-mercapto-4,5-dihydrothieno[3,2-e][1,2,4]triazolo[3,4-a]pyrimidin-5-one (5a)}

From $2 \mathrm{a}$ in $71 \%$ yield, m.p. 266-68 ${ }^{\circ} \mathrm{C}$ (dec.); IR (KBr) cm ${ }^{-1}: 2944(\mathrm{CH}$ alkyl), $1676(\mathrm{C}=\mathrm{O}), 1635$ $(\mathrm{C}=\mathrm{N}), 1583(\mathrm{C}=\mathrm{C}) ;{ }^{1} \mathrm{H}-\mathrm{NMR}$ (DMSO-d $)$ ppm: $\delta 2.17\left(\mathrm{~s}, 3 \mathrm{H}, \mathrm{CH}_{3}\right), \delta 2.22\left(\mathrm{~s}, 3 \mathrm{H}, \mathrm{CH}_{3}\right), \delta 2.44(\mathrm{~s}$, $1 \mathrm{H}, \mathrm{SH}), \delta 3.97\left(\mathrm{~s}, 3 \mathrm{H}, \mathrm{N}-\mathrm{CH}_{3}\right)$. Analyses: $\mathrm{C}_{10} \mathrm{H}_{10} \mathrm{~N}_{4} \mathrm{OS}_{2}$ (266.4). Required: $\mathrm{C}, 45.10 ; \mathrm{H}, 3.79 ; \mathrm{N}$, 21.04; Found: C, 45.33; H, 3.61; N, 21.21.

4-Methyl-1-mercapto-6,7,8,9-tetrahydrobenzo-4,5-dihydrothieno[3,2-e][1,2,4]triazolo[3,4-a]pyrimidin-5-one (5b)

From $2 \mathbf{b}$ in $71 \%$ yield, m.p. $233-35^{\circ} \mathrm{C}$ (dec.); IR (KBr) cm $\mathrm{cm}^{-1}$ :, 2931 (CH alkyl), $1680(\mathrm{C}=\mathrm{O}), 1632$ $(\mathrm{C}=\mathrm{N}), 1571(\mathrm{C}=\mathrm{C}) ;{ }^{1} \mathrm{H}-\mathrm{NMR}$ (DMSO-d $)$ ppm: $\delta 1.98\left(\mathrm{~m}, 4 \mathrm{H}, 2 \mathrm{CH}_{2}\right), \delta 2.53(\mathrm{~s}, 1 \mathrm{H}, \mathrm{SH}), \delta 2.94(\mathrm{t}$, $\left.2 \mathrm{H}, \mathrm{CH}_{2}\right), \delta 3.09$ (t, $2 \mathrm{H}, \mathrm{CH}_{2}$ ), $\delta 3.94\left(\mathrm{~s}, 3 \mathrm{H}, \mathrm{N}-\mathrm{CH}_{3}\right.$ ). Analyses: $\mathrm{C}_{12} \mathrm{H}_{12} \mathrm{~N}_{4} \mathrm{OS}_{2}$ (292.4). Required: $\mathrm{C}$, 49.29; H, 4.15; N, 19.17; Found: C, 49.20; H, 4.18; N, 19.28.

General procedure for the preperation of $\mathbf{6 a - f}$

A mixture of compound $\mathbf{2 a}$ or $\mathbf{2 b}(10 \mathrm{mmol})$, the appropriate aromatic aldehyde $(10 \mathrm{mmol})$ and anhydrous sodium acetate $(1.64 \mathrm{~g}, 20 \mathrm{mmol})$ was stirred under reflux in glacial acetic acid $(30 \mathrm{~mL})$ for 5 hr. The reaction mixture was allowed to cool to room temperature, poured into water (100 $\mathrm{mL})$, whereby the formed solid was filtered off and crystallized from an appropriate solvent to produce 6a-f in high yields. 


\section{3,5,6-Trimethyl-3,4-dihydrothieno[2,3-d]pyrimidin-4-one-2-benzaldehyde hydrazone (6a)}

From compound $2 \mathbf{a}(10 \mathrm{mmol})$ and benzaldehyde $(1.06 \mathrm{~g}, 10 \mathrm{mmol})$. The compound was obtained as pale white crystals, crystallized from acetic acid in $70 \%$ yield, m.p. $288-91^{\circ} \mathrm{C}$; IR $(\mathrm{KBr}) \mathrm{cm}^{-1}: 3373$ (brs, $\mathrm{NH}), 3047$ (CH aryl), 2917 (CH alkyl), $1675(\mathrm{C}=\mathrm{O}), 1625(\mathrm{C}=\mathrm{N}), 1586(\mathrm{C}=\mathrm{C}) ;{ }^{1} \mathrm{H}-\mathrm{NMR}$ (DMSO- $\left.d_{6}\right)$ ppm: $\delta 2.22\left(\mathrm{~s}, 3 \mathrm{H}, \mathrm{CH}_{3}\right), \delta 2.31\left(\mathrm{~s}, 3 \mathrm{H}, \mathrm{CH}_{3}\right), \delta 3.84\left(\mathrm{~s}, 3 \mathrm{H}, \mathrm{N}-\mathrm{CH}_{3}\right), \delta$ 7.21-7.75 (m,5H,phenyl protons), $\delta 8.89$ (s,1H,methylenic proton), $\delta 11.52$ (br,1H,NH, $\mathrm{D}_{2} \mathrm{O}$ exchangeable); Analyses: $\mathrm{C}_{16} \mathrm{H}_{16} \mathrm{~N}_{4} \mathrm{OS}$ (312.4) Requierd: C, 61.51; H, 5.16; N, 17.94; Found: C, 61.47; H, 4.99; N, 18.01.

\section{3,5,6-Trimethyl-3,4-dihydrothieno[2,3-d]pyrimidin-4-one-2-p-chlorobenzaldehyde hydrazone (6b)}

From compound 2a $(10 \mathrm{mmol})$ and 4-chlorobenzaldehyde $(1.41 \mathrm{~g}, 10 \mathrm{mmol})$. The compound was obtained as pale light yellow crystals, crystallized from dioxane in $72 \%$ yield, m.p. $278-80^{\circ} \mathrm{C}$ (dec.); IR $(\mathrm{KBr}) \mathrm{cm}^{-1}$ : 3250 (br, NH), 3040 (CH aryl), $2920(\mathrm{CH}$ alkyl), $1670(\mathrm{C}=\mathrm{O}), 1600(\mathrm{C}=\mathrm{N}), 1500(\mathrm{C}=\mathrm{C})$; ${ }^{1} \mathrm{H}-\mathrm{NMR}\left(\mathrm{DMSO}-d_{6}\right)$ ppm: $\delta 2.23\left(\mathrm{~s}, 3 \mathrm{H}, \mathrm{CH}_{3}\right), \delta 2.31\left(\mathrm{~s}, 3 \mathrm{H}, \mathrm{CH}_{3}\right), \delta 3.89\left(\mathrm{~s}, 3 \mathrm{H}, \mathrm{N}-\mathrm{CH}_{3}\right) \delta 7.62(\mathrm{dd}$, $4 \mathrm{H}$, phenyl protons), $\delta 8.84$ (s, $1 \mathrm{H}$, methylenic proton), $\delta 14.02$ (brs, $1 \mathrm{H}, \mathrm{NH}, \mathrm{D}_{2} \mathrm{O}$ exchangeable); ${ }^{13} \mathrm{C}$ NMR: 12.06, $12.56\left(2 \mathrm{CH}_{3}\right), 30.42\left(\mathrm{~N}_{-} \mathrm{CH}_{3}\right)$ 119.89-158.35 (12C, sp ${ }^{2}$ carbon atoms), $164.38(\mathrm{C}=\mathrm{O})$. Analyses: $\mathrm{C}_{16} \mathrm{H}_{15} \mathrm{ClN}_{4} \mathrm{OS}$ (346.9). Required: C, 55.40; H, 4.37; N, 16.15; Found: C, 55.21; H, 4.28; N, 16.32 .

\section{3,5,6-Trimethyl-3,4-dihydrothieno[2,3-d]pyrimidin-4-one-2-p-methoxybenzaldehyde hydrazone (6c)}

From compound 2a (10 mmol) and 4-methoxybenzaldehyde (1.36g, $10 \mathrm{mmol})$. The compound was obtained as pale white crystals, crystallized from dioxane in $68 \%$ yield, m.p. $246-49^{\circ} \mathrm{C}(\mathrm{dec}$.); IR (KBr) $\mathrm{cm}^{-1}$ : 3368 (brs, NH), 3044 (CH aryl), $2916\left(\mathrm{CH}\right.$ alkyl), $1676(\mathrm{C}=\mathrm{O}), 1605(\mathrm{C}=\mathrm{N}), 1517(\mathrm{C}=\mathrm{C}) ;{ }^{1} \mathrm{H}-$ NMR (DMSO- $d_{6}$ ) ppm: $\delta 2.25\left(\mathrm{~s}, 3 \mathrm{H}, \mathrm{CH}_{3}\right), \delta 2.30\left(\mathrm{~s}, 3 \mathrm{H}, \mathrm{CH}_{3}\right), \delta 3.79\left(\mathrm{~s}, 3 \mathrm{H}, \mathrm{N}_{-} \mathrm{CH}_{3}\right), \delta 3.93(\mathrm{~s}, 3 \mathrm{H}$, $\mathrm{OCH}_{3}$ ), $\delta 7.41$ (dd, $4 \mathrm{H}$, phenyl protons), $\delta 7.98$ (s, $1 \mathrm{H}$, methylenic proton), $\delta 11.48$ (brs, $1 \mathrm{H}, \mathrm{NH}, \mathrm{D}_{2} \mathrm{O}$ exchangeable). Analyses: $\mathrm{C}_{17} \mathrm{H}_{18} \mathrm{~N}_{4} \mathrm{O}_{2} \mathrm{~S}$ (342.5). Required: C, 59.61; H, 5.31; N, 16.38; Found: C, 59.37; H, 5.41; N, 16.19.

3-Methyl-5,6,7,8-tetrahydrobenzo-3,4-dihydrothieno[2,3d]pyrimidin-4-one-2-benzaldehyde hydrazone (6d)

From compound $\mathbf{2 b}(10 \mathrm{mmol})$ and benzaldehyde $(1.06 \mathrm{~g}, 10 \mathrm{mmol})$. The compound was obtained as pale white crystals, crystallized from acetic acid in $71 \%$ yield, m.p. $242-44^{\circ} \mathrm{C}$; IR ( $\left.\mathrm{KBr}\right) \mathrm{cm}^{-1}: 3370$ (brs, $\mathrm{NH}), 3047$ (CH aryl), 2919 (CH alkyl), $1674(\mathrm{C}=\mathrm{O}), 1615(\mathrm{C}=\mathrm{N}), 1596(\mathrm{C}=\mathrm{C}) ;{ }^{1} \mathrm{H}-\mathrm{NMR}$ (DMSO- $\left.d_{6}\right)$ ppm: $\delta 1.92\left(\mathrm{~m}, 4 \mathrm{H}, 2 \mathrm{CH}_{2}\right), \delta 2.91\left(\mathrm{t}, 2 \mathrm{H}, \mathrm{CH}_{2}\right), \delta 3.09\left(\mathrm{t}, 2 \mathrm{H}, \mathrm{CH}_{2}\right), \delta 3.94\left(\mathrm{~s}, 3 \mathrm{H}, \mathrm{N}-\mathrm{CH}_{3}\right), \delta$ 7.097.85 (m, 5H, phenyl protons), $\delta 8.45$ (s, $1 \mathrm{H}$, methylenic proton), $\delta 12.20$ (brs, $1 \mathrm{H}, \mathrm{NH}, \mathrm{D}_{2} \mathrm{O}$ exchangeable). Analyses: $\mathrm{C}_{18} \mathrm{H}_{18} \mathrm{~N}_{4} \mathrm{OS}$ (338.5). Requierd: C, 63.87; H, 5.37; N, 16.56; Found: C, 63.76; H, 5.19; $\mathrm{N}, 16.28$. 
3-Methyl-5,6,7,8-tetrahydrobenzo-3,4-dihydrothieno[2,3-d]pyrimidin-4-one-2-(p-chlorobenzalde-hyde hydrazone $(\mathbf{6 e})$

From compound $\mathbf{2 b}(10 \mathrm{mmol})$ and 4-chlorobenzaldehyde $(1.41 \mathrm{~g}, 10 \mathrm{mmol})$. The compound was obtained as pale light yellow crystals, crystallized from dioxane in $72 \%$ yield, m.p. $255-58^{\circ} \mathrm{C}$ (dec.); IR $(\mathrm{KBr}) \mathrm{cm}^{-1}$ : 3250 (brs, NH), 3040 (CH aryl), 2921 (CH alkyl), $1673(\mathrm{C}=\mathrm{O}), 1609(\mathrm{C}=\mathrm{N}), 1524(\mathrm{C}=\mathrm{C})$; ${ }^{1} \mathrm{H}-\mathrm{NMR}\left(\mathrm{DMSO}-d_{6}\right)$ ppm: $\delta 1.96\left(\mathrm{~m}, 4 \mathrm{H}, 2 \mathrm{CH}_{2}\right), \delta 2.94\left(\mathrm{t}, 2 \mathrm{H}, \mathrm{CH}_{2}\right), \delta 3.05\left(\mathrm{t}, 2 \mathrm{H}, \mathrm{CH}_{2}\right), \delta 3.94(\mathrm{~s}$, $3 \mathrm{H}, \mathrm{N}-\mathrm{CH}_{3}$ ), $\delta 7.65$ (dd, $4 \mathrm{H}$, phenyl protons), $\delta 8.44$ (s, $1 \mathrm{H}$, methylenic proton), $\delta 10.67$ (brs, $1 \mathrm{H}, \mathrm{NH}$, $\mathrm{D}_{2} \mathrm{O}$ exchangeable); ${ }^{13} \mathrm{C}-\mathrm{NMR}: 21.77-25.59\left(4 \mathrm{CH}_{2}\right), 30.19\left(\mathrm{~N}_{-} \mathrm{CH}_{3}\right)$ 118.54-158.89 (12C, sp ${ }^{2}$ carbon atoms), $161.35(\mathrm{C}=\mathrm{O})$. Analyses: $\mathrm{C}_{18} \mathrm{H}_{17} \mathrm{~N}_{4} \mathrm{ClOS}$ (372.9). Required: C, 57.97; H, 4.60; N, 15.03; Found: C, 57.81; H, 4.47; N, 14.79.

3-Methyl-5,6,7,8-tetrahydrobenzo-3,4-dihydrothieno[2,3-d]pyrimidin-4-one-2-(p-methoxybenzaldehyde hydrazone (6f)

From compound $\mathbf{2 b}(10 \mathrm{mmol})$ and 4-methoxybenzaldehyde $(1.36 \mathrm{~g}, 10 \mathrm{mmol})$. The compound was obtained as pale light white crystals, crystallized from dioxane in $68 \%$ yield, m.p. $262-64^{\circ} \mathrm{C}$ (dec.); IR $(\mathrm{KBr}) \mathrm{cm}^{-1}$ : 3368 (brs, NH), $3044(\mathrm{CH}$ aryl), $2916(\mathrm{CH}$ alkyl), $1676(\mathrm{C}=\mathrm{O}), 1605(\mathrm{C}=\mathrm{N}), 1517(\mathrm{C}=\mathrm{C})$; ${ }^{1} \mathrm{H}-\mathrm{NMR}\left(\mathrm{DMSO}-d_{6}\right)$ ppm: $\delta 1.93\left(\mathrm{~m}, 4 \mathrm{H}, 2 \mathrm{CH}_{2}\right), \delta 2.97\left(\mathrm{t}, 2 \mathrm{H}, \mathrm{CH}_{2}\right), \delta 3.01\left(\mathrm{t}, 2 \mathrm{H}, \mathrm{CH}_{2}\right), \delta 3.83(\mathrm{~s}$, $\left.3 \mathrm{H}, \mathrm{N}-\mathrm{CH}_{3}\right), \delta 3.95\left(\mathrm{~s}, 3 \mathrm{H}, \mathrm{OCH}_{3}\right), \delta 7.42$ (dd, $4 \mathrm{H}$, phenyl protons), $\delta 8.08$ (s, 1H, methylenic proton), $\delta 11.25$ (brs, $1 \mathrm{H}, \mathrm{NH}, \mathrm{D}_{2} \mathrm{O}$ exchangeable). Analyses: $\mathrm{C}_{19} \mathrm{H}_{20} \mathrm{~N}_{4} \mathrm{O}_{2} \mathrm{~S}$ (368.5). Required: C, 61.93; $\mathrm{H}$, 5.48; N, 15.21; Found: C, 61.88; H, 5.31; N, 15.7.

General procedure for the preparation of 7a-f

A mixture of compound 6a-f $(10 \mathrm{mmol})$, anhydrous sodium acetate $(1.64 \mathrm{~g}, 20 \mathrm{mmol})$ and bromine $(1.60 \mathrm{~g}, 10 \mathrm{mmol})$ was heated gently in glacial acetic acid $(30 \mathrm{~mL})$ in a water bath at $80^{\circ} \mathrm{C}$ for $16 \mathrm{hr}$. The reaction mixture was allowed to cool to room temperature, poured into water $(100 \mathrm{ml})$ and the solid soformed was collected by filtration and crystallized from appropriate solvent, to yield 7a-c.

\section{1-Phenyl-4,6,7-trimethyl-4,5-dihydrothieno[3,2-e][1,2,4]triazolo[3,4-a]pyrimidinone (7a)}

From compound $\mathbf{6 a}(10 \mathrm{mmol})$. The compound was obtained as yellow crystals, crystallized from dioxane in $62 \%$ yield, m.p. $328-30^{\circ} \mathrm{C}$ (dec.); IR ( $\left.\mathrm{KBr}\right) \mathrm{cm}^{-1}: 3049(\mathrm{CH}$ aryl), 2914 (CH alkyl), 1653 $(\mathrm{C}=\mathrm{O}), 1556(\mathrm{C}=\mathrm{N}), 1489(\mathrm{C}=\mathrm{C}) ;{ }^{1} \mathrm{H}-\mathrm{NMR}\left(\mathrm{DMSO}-d_{6}\right) \mathrm{ppm}: \delta 2.22\left(\mathrm{~s}, 3 \mathrm{H}, \mathrm{CH}_{3}\right), \delta 2.35\left(\mathrm{~s}, 3 \mathrm{H}, \mathrm{CH}_{3}\right)$, $\delta 3.89\left(\mathrm{~s}, 3 \mathrm{H}, \mathrm{N}-\mathrm{CH}_{3}\right) \delta$ 7.09-7.44 (m, 5H, phenyl protons); ${ }^{13} \mathrm{C}-\mathrm{NMR}$ (DMSO- $d_{6}$ ) ppm: 12.61, 12.83, $31.41\left(3 \mathrm{CH}_{3}\right), 118.26-159.24\left(12 \mathrm{C} \mathrm{sp}{ }^{2}\right.$ carbon atoms), $162.91(\mathrm{C}=\mathrm{O})$. Analyses: $\mathrm{C}_{16} \mathrm{H}_{14} \mathrm{~N}_{4} \mathrm{OS}(310.4)$. Required: C, 61.91; H, 4.56; N, 18.05; Found: C, 61.72; H, 4.41; N, 18.17. 
1-(4-Chlorophenyl)-4,6,7-trimethyl-4,5-dihydrothieno[3,2-e][1,2,4]triazolo[3,4-a]pyrimidin-5-one (7b)

From compound $\mathbf{6 b}(10 \mathrm{mmol})$. The compound was obtained as pale yellow crystals, crystallized from dioxane in 59\% yield, m.p. $302-4^{\circ} \mathrm{C}$ (dec.); IR (KBr) cm ${ }^{-1}: 3060(\mathrm{CH}$ aryl), $2920(\mathrm{CH}$ alkyl), 1687 $(\mathrm{C}=\mathrm{O}), 1611(\mathrm{C}=\mathrm{N}), 1556(\mathrm{C}=\mathrm{C}) ;{ }^{1} \mathrm{H}-\mathrm{NMR}\left(\mathrm{DMSO}-d_{6}\right) \mathrm{ppm}: \delta 2.35\left(\mathrm{~s}, 3 \mathrm{H}, \mathrm{CH}_{3}\right), \delta 2.45\left(\mathrm{~s}, 3 \mathrm{H}, \mathrm{CH}_{3}\right)$, $\delta 3.85$ (s, $3 \mathrm{H}, \mathrm{N}-\mathrm{CH}_{3}$ ), $\delta 7.65$ (dd, $4 \mathrm{H}$, phenyl protons); Analyses: $\mathrm{C}_{16} \mathrm{H}_{13} \mathrm{ClN}_{4} \mathrm{OS}$ (344.8). Required: C, 55.73 ; H, 3.81; N, 16.25; Found: C, 55.59; H, 3.71; N, 16.03.

\section{1-(4-Methoxyphenyl)-4,6,7-trimethyl-4,5-dihydrothieno[3,2-e][1,2,4]triazolo[3,4-a]pyrimidin-5-one} (7c)

From compound $\mathbf{6 c}(10 \mathrm{mmol})$. The compound was obtained as light yellow crystals, crystallized dioxane in $48 \%$ yield, m.p. $282-84^{\circ} \mathrm{C}$ (dec.); IR ( $\left.\mathrm{KBr}\right) \mathrm{cm}^{-1}$ : $3046(\mathrm{CH}$ aryl), 2916 (CH alkyl), 1664 $(\mathrm{C}=\mathrm{O}), 1557(\mathrm{C}=\mathrm{N}), 1499(\mathrm{C}=\mathrm{C}) ;{ }^{1} \mathrm{H}-\mathrm{NMR}\left(\mathrm{DMSO}-d_{6}\right) \mathrm{ppm}: \delta 2.33\left(\mathrm{~s}, 3 \mathrm{H}, \mathrm{CH}_{3}\right), \delta 2.41\left(\mathrm{~s}, 3 \mathrm{H}, \mathrm{CH}_{3}\right)$, $\delta 3.89$ (s, $3 \mathrm{H}, \mathrm{N}-\mathrm{CH}_{3}$ ), $\delta 7.14$ (dd, $4 \mathrm{H}$, phenyl protons); Analyses: $\mathrm{C}_{17} \mathrm{H}_{16} \mathrm{~N}_{4} \mathrm{O}_{2} \mathrm{~S}$ (340.4). Required: $\mathrm{C}$, 59.98; H, 4.75; N, 16.46; Found: C, 59.73; H, 4.52; N, 16.28.

4-Methyl-1-phenyl-6,7,8,9-tetrahydrobenzo-4,5-dihydrothieno[3,2-e][1,2,4]triazolo[3,4-b]pyrimi-din5 -one $(\mathbf{7 d})$

From compound $\mathbf{6 d}$ (10 mmol). The compound was obtained as yellow crystals, crystallized from dioxane in $52 \%$ yield, m.p. $272-74^{\circ} \mathrm{C}$ (dec.); IR ( $\left.\mathrm{KBr}\right) \mathrm{cm}^{-1}$ : 3049 (CH aryl), 2914 (CH alkyl), 1653 $(\mathrm{C}=\mathrm{O}), 1556(\mathrm{C}=\mathrm{N}), 1489(\mathrm{C}=\mathrm{C}) ;{ }^{1} \mathrm{H}-\mathrm{NMR}\left(\mathrm{DMSO}-d_{6}\right) \mathrm{ppm}: \delta 1.94\left(\mathrm{~m}, 4 \mathrm{H}, 2 \mathrm{CH}_{2}\right), \delta 2.90(\mathrm{t}, 2 \mathrm{H}$, $\left.\mathrm{CH}_{2}\right), \delta 3.19\left(\mathrm{t}, 2 \mathrm{H}, \mathrm{CH}_{2}\right), \delta 3.91\left(\mathrm{~s}, 3 \mathrm{H}, \mathrm{N}-\mathrm{CH}_{3}\right), \delta$ 7.21-7.56 (m, 5H, phenyl protons); ${ }^{13} \mathrm{C}-\mathrm{NMR}$ : 21.83-25.53 $\left(4 \mathrm{CH}_{2}\right), 29.89\left({\left.\mathrm{~N}-\mathrm{CH}_{3}\right)}^{2}\right.$ 118.34-158.96 (12C, sp ${ }^{2}$ carbon atoms), $163.30(\mathrm{C}=\mathrm{O})$; Analyses: $\mathrm{C}_{18} \mathrm{H}_{16} \mathrm{~N}_{4} \mathrm{OS}$ (336.4). Required: C, 64.26; H, 4.80; N, 16.66; Found: C, 64.02; H, 4.63; N, 16.49.

4-Methyl-1-(4-chlorophenyl)-6,7,8,9-tetrahydrobenzo-4,5-dihydrothieno[3,2-e][1,2,4triazolo[3,4-a]pyrimidin-5-one (7e)

From compound 6e $(10 \mathrm{mmol})$. The compound was obtained as pale yellow crystals, crystallized from dioxane in 59\% yield, m.p. $287-90^{\circ} \mathrm{C}$ (dec.); IR (KBr) $\mathrm{cm}^{-1}$ : $3060(\mathrm{CH}$ aryl), $2920(\mathrm{CH}$ alkyl), $1653(\mathrm{C}=\mathrm{O}), 1611(\mathrm{C}=\mathrm{N}), 1556(\mathrm{C}=\mathrm{C}) ;{ }^{1} \mathrm{H}-\mathrm{NMR}\left(\mathrm{DMSO}-d_{6}\right) \mathrm{ppm}: \delta 1.92\left(\mathrm{~m}, 4 \mathrm{H}, 2 \mathrm{CH}_{2}\right), \delta 2.93(\mathrm{t}$, $\left.2 \mathrm{H}, \mathrm{CH}_{2}\right), \delta 3.11\left(\mathrm{t}, 2 \mathrm{H}, \mathrm{CH}_{2}\right), \delta 3.86\left(\mathrm{~s}, 3 \mathrm{H}, \mathrm{N}-\mathrm{CH}_{3}\right), \delta 7.41$ (dd, $4 \mathrm{H}$, phenyl protons). Analyses: $\mathrm{C}_{18} \mathrm{H}_{15} \mathrm{ClN}_{4} \mathrm{OS}$ (370.9). Required: C, 58.29; H, 4.09; N, 15.11; Found: C, 58.22; H, 3.91; N, 14.98. 
4-Methyl-1-(4-methoxyphenyl)-6,7,8,9-tetrahydrobenzo-4,5-dihydrothieno[3,2-e][1,2,4]triazolo[3,4a]pyrimidin-5-one (7f)

From compound $\mathbf{6 f}(10 \mathrm{mmol})$. The compound was obtained as a light yellow crystals, crystallized from dioxane in 51\% yield, m.p. $301-303^{\circ} \mathrm{C}$ (dec.); IR (KBr) cm ${ }^{-1}: 3054(\mathrm{CH}$ aryl), $2916(\mathrm{CH}$ alkyl), $1664(\mathrm{C}=\mathrm{O}), 1557(\mathrm{C}=\mathrm{N}), 1499(\mathrm{C}=\mathrm{C})$. Analyses: $\mathrm{C}_{19} \mathrm{H}_{18} \mathrm{~N}_{4} \mathrm{SO}_{2}$ (366.5). Required: $\mathrm{C}, 62.26$; H, 4.96; N, 15.29; Found: C, 62.17; H, 4.88; N, 15.09.

General procedure for the preparation of $\mathbf{8 a}, \mathbf{b}$

A mixture of compound $\mathbf{2 a}$ or $\mathbf{2 b}(10 \mathrm{mmol})$ and ethyl acetoacetate $(1.30 \mathrm{~g}, 10 \mathrm{mmol})$ was refluxed in absolute ethanol $(30 \mathrm{~mL})$ for $5 \mathrm{hr}$. The reaction mixture was allowed to cool and the solid product so produced was filtered off and crystallized from ethanol to produce $\mathbf{8 a}$ or $\mathbf{8 b}$.

\section{2-Ethylacetoacetatehydrazone-3,5,6-trimethyl-3,4-dihydrothieno[2,3-d]pyrimidin-4-one (8a)}

From 2a in $85 \%$ yield, m.p. $149-52^{\circ} \mathrm{C}$; IR (KBr) cm ${ }^{-1}: 3156$ (brs, NH), 2949 (CH alkyl), 1730, 1672 $(2 \mathrm{C}=\mathrm{O}), 1567(\mathrm{C}=\mathrm{N}), 1511(\mathrm{C}=\mathrm{C}) ;{ }^{1} \mathrm{H}-\mathrm{NMR}\left(\mathrm{CDCl}_{3}\right) \mathrm{ppm}: \delta 1.27\left(\mathrm{t}, 3 \mathrm{H}, \mathrm{CH}_{3}\right), \delta 2.00\left(\mathrm{~s}, 3 \mathrm{H}, \mathrm{CH}_{3}\right), \delta$ $2.28\left(\mathrm{~s}, 3 \mathrm{H}, \mathrm{CH}_{3}\right), \delta 2.40\left(\mathrm{~s}, 3 \mathrm{H}, \mathrm{CH}_{3}\right), \delta 3.26\left(\mathrm{~s}, 2 \mathrm{H}, \mathrm{CH}_{2}\right), \delta 3.89\left(\mathrm{~s}, 3 \mathrm{H}, \mathrm{N}-\mathrm{CH}_{3}\right) \delta 4.19(\mathrm{q}, 2 \mathrm{H}$, $\mathrm{CH}_{2}$ ), $\delta 9.39$ (brs, $1 \mathrm{H}, \mathrm{NH}, \mathrm{D}_{2} \mathrm{O}$ exchangeable); ${ }^{13} \mathrm{C}-\mathrm{NMR}: 12.57,12.96,14.15,15.77,30.23\left(5 \mathrm{CH}_{3}\right)$, 44.32, $61.29\left(2 \mathrm{CH}_{2}\right), 118.08-158.37\left(6 \mathrm{C} \mathrm{sp}{ }^{2}\right.$ carbon atoms), 164.72, $169.31(2 \mathrm{C}=\mathrm{O})$. Analyses: $\mathrm{C}_{15} \mathrm{H}_{20} \mathrm{~N}_{4} \mathrm{O}_{3} \mathrm{~S}$ (336.5). Required: C, 53.54; H, 5.99; N, 16.65; Found: C, 53.39; H, 5.67; N, 16.47.

2-Ethylacetoacetatehydrazone-5,6,7,8-tetrahydrobenzo-3-methyl-3,4-dihydrothieno[2,3-d] pyrimidin4-one (8b)

From 2b in 85\% yield, m.p. $162-64^{\circ} \mathrm{C}$; IR (KBr) cm ${ }^{-1}: 3150$ (brs, NH), 2960 (CH alkyl), 1740, 1680 $(2 \mathrm{C}=\mathrm{O}), 1580(\mathrm{C}=\mathrm{N}), 1500(\mathrm{C}=\mathrm{C}) ;{ }^{1} \mathrm{H}-\mathrm{NMR}\left(\mathrm{CDCl}_{3}\right) \mathrm{ppm}: \delta 1.29\left(\mathrm{t}, 3 \mathrm{H}, \mathrm{CH}_{3}\right), \delta 1.96\left(\mathrm{~m}, 4 \mathrm{H}, 2 \mathrm{CH}_{2}\right)$, $\delta 2.90\left(\mathrm{t}, 2 \mathrm{H}, \mathrm{CH}_{2}\right), \delta 3.11\left(\mathrm{t}, 2 \mathrm{H}, \mathrm{CH}_{2}\right), \delta 3.22\left(\mathrm{~s}, 2 \mathrm{H}, \mathrm{CH}_{2}\right), \delta 3.80\left(\mathrm{~s}, 3 \mathrm{H}, \mathrm{N}-\mathrm{CH}_{3}\right), \delta 4.13(\mathrm{q}, 2 \mathrm{H}$, $\mathrm{CH}_{2}$ ), $\delta$ 10.31(brs, $1 \mathrm{H}, \mathrm{NH}, \mathrm{D}_{2} \mathrm{O}$ exchangeable); Analyses: $\mathrm{C}_{17} \mathrm{H}_{22} \mathrm{~N}_{4} \mathrm{SO}_{3}$ (362.5). Required: C, 56.34; H, 6.13; N, 15.46; Found: C, 56.21; H, 6.06; N, 15.39.

General procedure for the preparation of $\mathbf{9 a}, \mathbf{b}$

Method (A): A solution of compound $\mathbf{2 a}$ or $\mathbf{2 b}(10 \mathrm{mmol})$ and ethyl acetoacetate $(1.30 \mathrm{~g}, 10 \mathrm{mmol})$ was stirred under reflux in absolute ethanol $(30 \mathrm{~mL})$ for $30 \mathrm{hr}$. The reaction mixture was allowed to cool to room temperature, poured into cold water $(100 \mathrm{~mL})$. The deposited precipitate was filtered off, dried and crystallized from dioxane.

Method (B): A solution of compound $\mathbf{2 a}$ or $\mathbf{2 b}(10 \mathrm{mmol})$ and ethyl acetoacetate $(1.30 \mathrm{~g}, 10 \mathrm{mmol})$ 
in sodium ethoxide solution [prepared by dissolving sodium metal $(0.23 \mathrm{~g}, 10 \mathrm{mmol})$ in absolute ethanol $(30 \mathrm{~mL})$ ] was heated under reflux with stirring for $6 \mathrm{hr}$. The reaction mixture was allowed to cool and poured into cold water $(100 \mathrm{~mL})$ and neutralized by acetic acid, whereby a solid was precipitated, which was filtered off and crystallized from dioxane.

Method $(C)$ : A solution of compound $\mathbf{2 a}$ or $\mathbf{2 b}(10 \mathrm{mmol})$ was heated under reflux with sodium ethoxide solution [sodium metal $(0.23 \mathrm{~g}, 10 \mathrm{mmol})$ in absolute ethanol $(30 \mathrm{ml})$ ] for $3 \mathrm{hr}$. The reaction mixture was allowed to cool, poured into water $(100 \mathrm{~mL})$, neutralized with acetic acid, and the precipitate formed was filtered off and crystallized from dioxane.

\section{5,6-Dimethyl-2-(3-methyl-4H,5H-pyrazol-5-one-1-yl)-3,4-dihydrothieno[2,3-d]pyrimidin-4-one (9a)}

From 8a, in $72 \%$ yield, m.p. $219-22^{\circ} \mathrm{C}$; IR ( $\left.\mathrm{KBr}\right) \mathrm{cm}^{-1}: 2940(\mathrm{CH}$ alkyl), 1688, $1666(2 \mathrm{C}=\mathrm{O}), 1550$ $(\mathrm{C}=\mathrm{N}), 1500(\mathrm{C}=\mathrm{C}) ;{ }^{1} \mathrm{H}-\mathrm{NMR}\left(\mathrm{DMSO}-d_{6}\right)$ ppm: $\delta 2.30-2.33\left(\mathrm{~m}, 9 \mathrm{H}, 3 \mathrm{CH}_{3}\right), \delta 2.52\left(\mathrm{~s}, 2 \mathrm{H}, \mathrm{CH}_{2}\right), \delta$ 3.89 (s, 3H, N-CH 3 ). Analyses: $\mathrm{C}_{13} \mathrm{H}_{14} \mathrm{~N}_{4} \mathrm{O}_{2} \mathrm{~S}$ (290.4). Required: C, 53.77; H, 4.87; N, 19.30; Found: C, $53.65 ; \mathrm{H}, 4.52 ; \mathrm{N}, 19.07$.

\section{5,6,7,8-Tetrahydrobenzo-2-(3-methyl-4H,5H-pyrazol-5-one-1-yl)-3-methylthieno[2,3-d]pyrimidin-4-} one $(\mathbf{9 b})$

From 8b, in 72\% yield, m.p. 232-35 ${ }^{\circ} \mathrm{C}$; IR (KBr) cm ${ }^{-1}: 2940(\mathrm{CH}$ alkyl), 1670, $1600(2 \mathrm{C}=\mathrm{O}), 1550$ $(\mathrm{C}=\mathrm{N}), 1500(\mathrm{C}=\mathrm{C}) ;{ }^{1} \mathrm{H}-\mathrm{NMR}\left(\mathrm{DMSO}-d_{6}\right) \mathrm{ppm}: \delta 1.91\left(\mathrm{~m}, 4 \mathrm{H}, 2 \mathrm{CH}_{2}\right), \delta 2.23\left(\mathrm{~s}, 3 \mathrm{H}, \mathrm{CH}_{3}\right), \delta 2.52(\mathrm{~s}$, $\left.2 \mathrm{H}, \mathrm{CH}_{2}\right), \delta 2.90\left(\mathrm{t}, 2 \mathrm{H}, \mathrm{CH}_{2}\right), \delta 3.11\left(\mathrm{t}, 2 \mathrm{H}, \mathrm{CH}_{2}\right), \delta 3.90\left(\mathrm{~s}, 3 \mathrm{H}, \mathrm{N}-\mathrm{CH}_{3}\right)$; Analyses: $\mathrm{C}_{15} \mathrm{H}_{16} \mathrm{~N}_{4} \mathrm{O}_{2} \mathrm{~S}$ (316.4). Required: C, 56.94; H, 5.11; N, 17.71; Found: C, 56.88; H, 4.92; N, 17.56.

General procedure for the preparation of $\mathbf{1 0 a}, \mathbf{b}$

To a warmed ethanolic sodium ethoxide solution [prepared by dissolving sodium metal $(0.23 \mathrm{~g}, 10$ mmol in absolute ethanol $(30 \mathrm{~mL})$ ] was added either compound $\mathbf{2 a}$ or $\mathbf{2 b}(10 \mathrm{mmol})$ and ethyl cyanoacetate $(1.13 \mathrm{~g}, 10 \mathrm{mmol})$. The mixture was stirred under reflux for $8 \mathrm{hr}$, the reaction mixture was allowed to cool to room temperature, then poured into cold water $(100 \mathrm{~mL})$ and neutralized with acetic acid. The solid product was filtered off, washed with water, ethanol, dried and crystallized from dioxane.

\section{5,6-Dimethyl-2-(3-amino-4H,5H-5-pyrazolinon-1-yl)-3-methylthieno[2,3-d]pyrimidin-4-one (10a)}

From $2 \mathbf{a}$ in $38 \%$ yield, m.p.334- $7^{\circ} \mathrm{C}$ (dec.); IR (KBr) cm ${ }^{-1}: 3265$ (brs, NH), 2939 (CH alkyl), 1698 $(\mathrm{C}=\mathrm{O}), 1609(\mathrm{C}=\mathrm{N}), 1527(\mathrm{C}=\mathrm{C}) .{ }^{1} \mathrm{H}-\mathrm{NMR}\left(\mathrm{DMSO}-d_{6}\right)$ ppm: $\delta 2.32\left(\mathrm{~s}, 3 \mathrm{H}, \mathrm{CH}_{3}\right), \delta 2.42\left(\mathrm{~s}, 3 \mathrm{H}, \mathrm{CH}_{3}\right)$, $\delta 3.42\left(\mathrm{~s}, 2 \mathrm{H}, \mathrm{CH}_{2}\right), \delta 3.73\left(\mathrm{~s}, 3 \mathrm{H}, \mathrm{N}-\mathrm{CH}_{3}\right), \delta 12.3$ (brs, $\mathrm{NH}_{2}, \mathrm{D}_{2} \mathrm{O}$ exchangeable); ${ }^{13} \mathrm{C}-\mathrm{NMR}: 12.72$ $\left(\mathrm{CH}_{3}\right), 12.93\left(\mathrm{CH}_{3}\right), 14.31\left(\mathrm{CH}_{2}\right), 37.58\left(\mathrm{~N}-\mathrm{CH}_{3}\right), 118.01-153.27$ (6C sp ${ }^{2}$ carbon atoms), 159.32, 161.23 (2C=O). Analyses: $\mathrm{C}_{12} \mathrm{H}_{13} \mathrm{~N}_{5} \mathrm{O}_{2} \mathrm{~S}$ (291.4). Required: C, 49.46; H, 4.51; N, 24.04; Found: C, 49.51; H, 4.32; N, 24.12. 
5,6,7,8-Tetrahydrobenzo-2-(3-amino-4H,5H-5-pyrazolinon-1-yl)-3-methyl-thieno[2,3-d] pyrimidin-4one $(\mathbf{1 0 b})$

From $2 \mathbf{b}$ in $43 \%$ yield, m.p. $292-4^{\circ} \mathrm{C}$ (dec.); IR (KBr) cm ${ }^{-1}: 3219$ (br, NH), 2927 (CH alkyl), 1688 $(\mathrm{C}=\mathrm{O}), 1602(\mathrm{C}=\mathrm{N}), 1521(\mathrm{C}=\mathrm{C}){ }^{1} \mathrm{H}-\mathrm{NMR}\left(\mathrm{DMSO}-d_{6}\right) \mathrm{ppm}: \delta 1.92\left(\mathrm{~m}, 4 \mathrm{H}, 2 \mathrm{CH}_{2}\right), \delta 2.82(\mathrm{t}, 2 \mathrm{H}$, $\left.\mathrm{CH}_{2}\right), \delta 3.16\left(\mathrm{t}, 2 \mathrm{H}, \mathrm{CH}_{2}\right), \delta 3.23\left(\mathrm{~s}, 2 \mathrm{H}, \mathrm{CH}_{2}\right), \delta 3.76\left(\mathrm{~s}, 3 \mathrm{H}, \mathrm{N}-\mathrm{CH}_{3}\right), \delta 10.31$ (br, $\mathrm{NH}_{2}, \mathrm{D}_{2} \mathrm{O}$ exchangeable); Analyses: $\mathrm{C}_{14} \mathrm{H}_{15} \mathrm{~N}_{5} \mathrm{O}_{2} \mathrm{~S}$ (317.4). Required: C, 52.97; H, 4.77; N, 22.07; Found: C, 53.07; H, 4.59; N, 22.19 .

General procedure for the preparation of $\mathbf{1 1 a}, \mathbf{b}$

A mixture of compound $\mathbf{2 a}$ or $\mathbf{2 b}(10 \mathrm{mmol})$ with chloroacetone or phenacyl bromide $(10 \mathrm{mmol})$ was heated under reflux $5 \mathrm{hr}$ in dry xylene $(30 \mathrm{~mL})$. The solid precipitated that separated upon cooling was filtered off and crystallized from appropriate solvent to produce 11a,b in high yield .

\section{1,5,7,8-Tetramethyl-5,6-dihydrothieno[2‘,3`6,5]pyrimido[2,1-c][1,2,4]triazin-6-one (11a)}

From compound 2a $(10 \mathrm{mmol})$ and chloroacetone $(0.93 \mathrm{~g}, 10 \mathrm{mmol})$. The compound was obtained as pale white crystals, crystallized from ethanol in $51 \%$ yield, m.p. $257-59^{\circ} \mathrm{C}$ (dec.); IR (KBr) cm ${ }^{-1}: 3360$ (brs, NH), $2950\left(\mathrm{CH}\right.$ alkyl), $1689(\mathrm{C}=\mathrm{O}), 1600(\mathrm{C}=\mathrm{N}), 1550(\mathrm{C}=\mathrm{C}) ;{ }^{1} \mathrm{H}-\mathrm{NMR}$ (DMSO- $\left.d_{6}\right)$ ppm: $\delta 2.23$ $\left(\mathrm{s}, 3 \mathrm{H}, \mathrm{CH}_{3}\right), \delta 2.31\left(\mathrm{~s}, 3 \mathrm{H}, \mathrm{CH}_{3}\right), \delta 2.41\left(\mathrm{~s}, 3 \mathrm{H}, \mathrm{CH}_{3}\right), \delta 3.62\left(\mathrm{~s}, 3 \mathrm{H}, \mathrm{N}-\mathrm{CH}_{3}\right), \delta 4.38$ (brs, $1 \mathrm{H}, \mathrm{NH}, \mathrm{D}_{2} \mathrm{O}$ exchangeable), $\delta 9.02$ (s,1H,triazine). Analyses: $\mathrm{C}_{12} \mathrm{H}_{14} \mathrm{~N}_{4} \mathrm{OS}$ (262.4). Required: C, 54.93; H, 5.39; N, 21.36; Found: C, 55.11; H, 5.29; N, 21.07.

\section{1-Phenyl-5,7,8-trimethyl-5,6-dihydrothieno[2‘,3 :6,5]pyrimido[2,1-c][1,2,4] triazin-6-one (11b)}

From compound 2a $(10 \mathrm{mmol})$ and phenacylbromide $(1.99 \mathrm{~g}, 10 \mathrm{mmol})$. The compound was obtained as pale white crystals, crystallized from ethanol in $45 \%$ yield, m.p. $279-81^{\circ} \mathrm{C}$ (dec.); IR ( $\left.\mathrm{KBr}\right) \mathrm{cm}^{-1}$ : 3400 (brs, NH), 2958 (CH alkyl), $1694(\mathrm{C}=\mathrm{O}), 1589(\mathrm{C}=\mathrm{N}), 1559(\mathrm{C}=\mathrm{C})$; ${ }^{1} \mathrm{H}-\mathrm{NMR}$ (DMSO- $\left.d_{6}\right)$ ppm: $\delta$ $2.24\left(\mathrm{~s}, 3 \mathrm{H}, \mathrm{CH}_{3}\right), \delta 2.35\left(\mathrm{~s}, 3 \mathrm{H}, \mathrm{CH}_{3}\right), \delta 3.74\left(\mathrm{~s}, 3 \mathrm{H}, \mathrm{N}-\mathrm{CH}_{3}\right), \delta 7.02-7.84(\mathrm{~m}, 5 \mathrm{H}$, phenyl), $\delta 9.16$ (s, $1 \mathrm{H}$, triazine), $\delta 10.38$ (brs, $1 \mathrm{H}, \mathrm{NH}, \mathrm{D}_{2} \mathrm{O}$ exchangeable). Analyses: $\mathrm{C}_{17} \mathrm{H}_{16} \mathrm{~N}_{4} \mathrm{OS}$ (324.4). Required: $\mathrm{C}$, 62.94; H, 4.98; N, 17.28; Found: C, 62.78; H, 4.81; N, 16.86.

General procedure for the preparation of 12a-f

A mixture of compound $\mathbf{2 a}$ or $\mathbf{2 b}(10 \mathrm{mmol})$ and a $\beta$-diketone $(10 \mathrm{mmol})$ in absolute ethanol (30 $\mathrm{mL}$ ) was stirred under reflux for $5 \mathrm{hr}$. The reaction mixture was allowed to cool to $0^{\circ} \mathrm{C}$ for 3 hours. The precipitate was filtered off, dried and crystallized from the appropriate solvent to produce 12a-f in high yields. 


\section{3,5,6-Trimethyl-2-(3,5-dimethylpyrazolyl)-3,4-dihydrothieno[2,3-d]pyrimidin-4-one (12a)}

From compound 2a $(10 \mathrm{mmol})$ and pentane-2,4-dione $(1.00 \mathrm{~g}, 10 \mathrm{mmol})$. The compound was obtained as pale light crystals, crystallized from dioxane in $83 \%$ yield, m.p. $217-19^{\circ} \mathrm{C}$; IR $(\mathrm{KBr}) \mathrm{cm}^{-1}$ : $3060\left(\mathrm{CH}\right.$ aryl), $2960\left(\mathrm{CH}\right.$ alkyl), $1700(\mathrm{C}=\mathrm{O}), 1600(\mathrm{C}=\mathrm{N}), 1550(\mathrm{C}=\mathrm{C}) ;{ }^{1} \mathrm{H}-\mathrm{NMR}\left(\mathrm{DMSO}-d_{6}\right)$ ppm: $\delta$ $2.28\left(\mathrm{~s}, 3 \mathrm{H}, \mathrm{CH}_{3}\right), \delta 2.32\left(\mathrm{~s}, 3 \mathrm{H}, \mathrm{CH}_{3}\right), \delta 2.41\left(\mathrm{~s}, 3 \mathrm{H}, \mathrm{CH}_{3}\right), \delta 2.51\left(\mathrm{~s}, 3 \mathrm{H}, \mathrm{CH}_{3}\right), \delta 3.42(\mathrm{~s}, 3 \mathrm{H}, \mathrm{N}-$ $\left.\mathrm{CH}_{3}\right), \delta 6.01\left(\mathrm{~s}, 1 \mathrm{H}\right.$, pyrazole); ${ }^{13} \mathrm{C}-\mathrm{NMR}$ (DMSO- $\left.d_{6}\right): 11.53,1292,13.16,13.60\left(4 \mathrm{CH}_{3}\right), 31.69(\mathrm{~N}-$ $\mathrm{CH}_{3}$ ), 107.53-151.42 (8C sp ${ }^{2}$ carbon atoms), $159.24(\mathrm{C}=\mathrm{O})$. Analyses: $\mathrm{C}_{14} \mathrm{H}_{16} \mathrm{~N}_{4} \mathrm{OS}$ (288.4). Required: C, 58.31; H, 5.60; N, 19.43; Found: C, 57.97; H, 5.41; N, 19,26.

\section{3,5,6-Trimethyl-2-(3,5-dimethyl-4-chloropyrazolyl)-3,4-dihydrothieno[2,3-d]pyrimidin-4-one (12b)}

From compound 2a (10 mmol) and 3-chloropentane-2,4-dione (1.34g, $10 \mathrm{mmol})$. The compound was obtained as light white crystals, crystallized from DMF in $92 \%$ yield, m.p. $259-61^{\circ} \mathrm{C}$ (dec.); IR $(\mathrm{KBr}) \mathrm{cm}^{-1}: 2960\left(\mathrm{CH}\right.$ alkyl), $1680(\mathrm{C}=\mathrm{O}), 1600(\mathrm{C}=\mathrm{N}), 1580(\mathrm{C}=\mathrm{C}) ;{ }^{1} \mathrm{H}-\mathrm{NMR}\left(\mathrm{CDCl}_{3}\right)$ ppm: $\delta 2.29$ $\left(\mathrm{s}, 3 \mathrm{H}, \mathrm{CH}_{3}\right), \delta 2.34\left(\mathrm{~s}, 3 \mathrm{H}, \mathrm{CH}_{3}\right), \delta 2.41\left(\mathrm{~s}, 3 \mathrm{H}, \mathrm{CH}_{3}\right), \delta 2.51\left(\mathrm{~s}, 3 \mathrm{H}, \mathrm{CH}_{3}\right), \delta 3.44\left(\mathrm{~s}, 3 \mathrm{H}, \mathrm{N}-\mathrm{CH}_{3}\right)$; ${ }^{13} \mathrm{C}-\mathrm{NMR}\left(\mathrm{CDCl}_{3}\right): 10.23,11.56,12.91,13.19\left(4 \mathrm{CH}_{3}\right), 31.81\left(\mathrm{~N}_{-} \mathrm{CH}_{3}\right), 111.26-148.67\left(7 \mathrm{C} \mathrm{sp}^{2}\right.$ carbon atoms), $158.96(\mathrm{C}-\mathrm{Cl}), 159.13(\mathrm{C}=\mathrm{O})$. Analyses: $\mathrm{C}_{14} \mathrm{H}_{15} \mathrm{ClN}_{4} \mathrm{OS}$ (322.8). Required: $\mathrm{C}, 52.15 ; \mathrm{H}, 4.69$; N, 17.36; Found: C, 51.89; H, 4.45; N, 17.25.

3,5,6-Trimethyl-2-(3-methyl-5-trifluromethylpyrazolyl)-3,4-dihydrothieno[2,3-d]pyrimidin-4-one (12c)

From compound $2 \mathbf{a}(10 \mathrm{mmol})$ and 1,1,1-trifluro-2,4-pentanedione (1.54g, $10 \mathrm{mmol})$. The compound was obtained as a pale light colorless crystals, crystallized from ethanol in $82 \%$ yield, m.p. 231$33^{\circ} \mathrm{C}$; IR (KBr) cm IR $^{-1} 2980\left(\mathrm{CH}\right.$ alkyl), $1680(\mathrm{C}=\mathrm{O}), 1600(\mathrm{C}=\mathrm{N}), 1560(\mathrm{C}=\mathrm{C}) ;{ }^{1} \mathrm{H}-\mathrm{NMR}$ $\left(\mathrm{CDCl}_{3}:\right.$ DMSO- $\left.d_{6} / 4: 1\right)$ ppm: $\delta 2.10\left(\mathrm{~s}, 3 \mathrm{H}, \mathrm{CH}_{3}\right), \delta 2.35\left(\mathrm{~s}, 3 \mathrm{H}, \mathrm{CH}_{3}\right), \delta 2.451\left(\mathrm{~s}, 3 \mathrm{H}, \mathrm{CH}_{3}\right), \delta 2.56(\mathrm{~s}$, $\left.3 \mathrm{H}, \mathrm{N}-\mathrm{CH}_{3}\right), \delta 8.32$ (s, $1 \mathrm{H}$, pyrazole); ${ }^{13} \mathrm{C}-\mathrm{NMR}\left(\mathrm{CDCl}_{3}: \mathrm{DMSO}-d_{6} / 4: 1\right): 11.53,1292,13.16,13.60$ $\left(4 \mathrm{CH}_{3}\right), \quad 31.69\left(\mathrm{~N}-\mathrm{CH}_{3}\right), \quad 107.53-151.42\left(8 \mathrm{C} \quad \mathrm{sp}^{2}\right.$ carbon atoms), $159.24 \quad(\mathrm{C}=\mathrm{O})$. Analyses: $\mathrm{C}_{14} \mathrm{H}_{13} \mathrm{~F}_{3} \mathrm{~N}_{4} \mathrm{OS}$ (342.4). Required: C, 49.11; H, 3.83; N, 16.37; Found: C, 49.02; H, 3.71; N, 16.18.

3-Methyl-5,6,7,8-tetrahydrobenzo-2-(3,5-dimethylpyrazolyl)3,4-dihydrothieno[2,3-d]pyrimidin-4-one (12d)

From compound $\mathbf{2 b}(10 \mathrm{mmol})$ and pentane-2,4-dione $(1.00 \mathrm{~g}, 10 \mathrm{mmol})$. The compound was obtained as pale yellow crystals, crystallized from dioxane/ethanol in $71 \%$ yield, m.p. $248-50^{\circ} \mathrm{C}$ (dec.); IR $(\mathrm{KBr}) \mathrm{cm}^{-1}: 3060\left(\mathrm{CH}\right.$ aryl), $2960\left(\mathrm{CH}\right.$ alkyl), $1700(\mathrm{C}=\mathrm{O}), 1600(\mathrm{C}=\mathrm{N}), 1550(\mathrm{C}=\mathrm{C})$; ${ }^{1} \mathrm{H}-\mathrm{NMR}$ $\left(\mathrm{DMSO}-d_{6}\right)$ ppm: $\delta 1.87\left(\mathrm{~m}, 4 \mathrm{H}, 2 \mathrm{CH}_{2}\right), \delta 2.25\left(\mathrm{~s}, 3 \mathrm{H}, \mathrm{CH}_{3}\right), \delta 2.28\left(\mathrm{~s}, 3 \mathrm{H}, \mathrm{CH}_{3}\right), \delta 2.76(\mathrm{~m}, 2 \mathrm{H}$, $\left.\mathrm{CH}_{2}\right), \delta 3.07\left(\mathrm{~m}, 2 \mathrm{H}, \mathrm{CH}_{2}\right), \delta 3.41\left(\mathrm{~s}, 3 \mathrm{H}, \mathrm{N}-\mathrm{CH}_{3}\right)$ and $\delta 6.06\left(\mathrm{~s}, 1 \mathrm{H}\right.$, pyrazole). Analyses: $\mathrm{C}_{16} \mathrm{H}_{18} \mathrm{~N}_{4} \mathrm{OS}$ (314.4). Required: C, 61.12; H, 5.78; N, 17.82; Found: C, 61.09; H, 5.63; N, 17,56. 
3-Methyl-5,6,7,8-tetrahydrobenzo-2-(3,5-dimethyl-4-chloropyrazolyl)-3,4-dihydrothieno[2,3-d]pyrimidin-4-one (12e)

From compound $\mathbf{2 b}(10 \mathrm{mmol})$ and 3-chloropentane-2,4-dione $(1.34 \mathrm{~g}, 10 \mathrm{mmol})$. The compound was obtained as yellow crystals, crystallized from dimethylformamide in $81 \%$ yield, m.p. $278-80^{\circ} \mathrm{C}$ (dec.); IR (KBr) cm ${ }^{-1}: 2960\left(\mathrm{CH}\right.$ alkyl), $1689(\mathrm{C}=\mathrm{O}), 1608(\mathrm{C}=\mathrm{N}), 1580(\mathrm{C}=\mathrm{C}) ;{ }^{1} \mathrm{H}-\mathrm{NMR}\left(\mathrm{CDCl}_{3}\right)$ ppm: $\delta 1.88\left(\mathrm{~m}, 4 \mathrm{H}, 2 \mathrm{CH}_{2}\right), \delta 2.29\left(\mathrm{~s}, 3 \mathrm{H}, \mathrm{CH}_{3}\right), \delta 2.33\left(\mathrm{~s}, 3 \mathrm{H}, \mathrm{CH}_{3}\right), \delta 2.79\left(\mathrm{~m}, 2 \mathrm{H}, \mathrm{CH}_{2}\right), \delta 3.04(\mathrm{~m}$, $2 \mathrm{H}, \mathrm{CH}_{2}$ ) and $\delta 3.44$ (s, 3H, N-CH ). Analyses: $\mathrm{C}_{16} \mathrm{H}_{17} \mathrm{ClN}_{4} \mathrm{OS}$ (348.9). Required: $\mathrm{C}, 55.09 ; \mathrm{H}, 4.92 ; \mathrm{N}$, 16.06; Found: C, 55.18; H, 4.79; N, 15.98.

3-Methyl-5,6,7,8-tetrahydrobenzo-2-(3-methyl-5-trifluromethylpyrazolyl)-thieno[2,3-d]pyrimidin-4one (12f)

From compound $\mathbf{2 b}(10 \mathrm{mmol})$ and 1,1,1-trifluro-2,4-pentanedione (1.54g, $10 \mathrm{mmol})$. The compound was obtained as pale white crystals, crystallized from ethanol in $76 \%$ yield, m.p. $293-95^{\circ} \mathrm{C}$ (dec.); IR (KBr) cm ${ }^{-1}: 2980\left(\mathrm{CH}\right.$ alkyl), $1680(\mathrm{C}=\mathrm{O}), 1600(\mathrm{C}=\mathrm{N}), 1560(\mathrm{C}=\mathrm{C}) ;{ }^{1} \mathrm{H}-\mathrm{NMR}\left(\mathrm{CDCl}_{3}: \mathrm{DMSO}-d_{6} /\right.$ 4:1) ppm: $\delta 1.85\left(\mathrm{~m}, 4 \mathrm{H}, 2 \mathrm{CH}_{2}\right), \delta 2.11\left(\mathrm{~s}, 3 \mathrm{H}, \mathrm{CH}_{3}\right), \delta 2.72\left(\mathrm{~m}, 2 \mathrm{H}, \mathrm{CH}_{2}\right), \delta 2.99\left(\mathrm{~m}, 2 \mathrm{H}, \mathrm{CH}_{2}\right), \delta$ $3.56\left(\mathrm{~s}, 3 \mathrm{H}, \mathrm{N}-\mathrm{CH}_{3}\right)$ and $\delta 8.32\left(\mathrm{~s}, 1 \mathrm{H}\right.$, pyrazole). Analyses: $\mathrm{C}_{16} \mathrm{H}_{15} \mathrm{~F}_{3} \mathrm{~N}_{4} \mathrm{OS}$ (368.4). Required: $\mathrm{C}, 52.17$; H, 4.11; N, 15.21; Found: C, 52.01; H, 3.99; N, 15.03.

\section{References and Notes}

1. Pathak, U.S.; Singh, S.; Padh, J. Indian J. Chem., Section B 1991, 30, 618.

2. Shishoo, C. J.; Devani, M.B.; Bhadti, V.S.; Jain, K.S.; Rathod, I.S.; Goyal, R. K.; Gandhi, T.P.; Patel; R. B.; Naik, S. R. Arzneim-Forsch 1990, 40, 567.

3. Sugiyama, M.; Sakamoto, T.; Tabata, K.; Endo, K.; Ito, K.; Kobayashi, M.; Fukumi, H. Chem. Pharm. Bull. 1989, 37, 2122.

4. Kienzle, F.; Kaiser, A.; Minder, R. E. Helv. Chim. Acta 1983, 66, 48.

5. Jordis, U.; Sauter, F.; Siddiqi, S. M. Vest. Slov. Kem. Drus 1986, 33, 217.

6. Darias, V.; Abdallah, S. S.; Tello, M. L.; Delgado, L. D.; Vega, S. Arch. Pharm. (Weinheim, Ger.) 1994, 327, 779.

7. Carclunescu, D.; Lopez, A. D.; Iriarte, E. G.; Tina, G.; Gomez, G.; Tena, R.; Ghirvu, C. An. $R$. Acad. Farm. 1985, 51, 241.

8. Newberry, R. A.; Bushell, B. J. U.S. 3, 979, 40207 Sep 1976; Brit. Appl. 748, 870, 27 Feb. 1974 (C. A. 86:29795z).

9. Shishoo, C. J.; Jain, K. S. J. Heterocyclic Chem. 1992, 29, 883.

10. Clark, J.; Shahhet, M.S.; Korakas, D.; Varvounis, G. J. Heterocyclic Chem. 1993, 30, 1065.

11. Sauter, F.; Deinhammer, W.; Stanetty, P. Monatsh. Chem. 1974, 105, 1258. 
12. Abdel-Fattah, A. M.; Aly, A. S.; Gad, F. A.; Zaki, M. E. A.; El-Gazzar, A. B. A. Phosphorus, Sulfur and Silicon 1998, 141, 263.

13. Abdel-Fattah, A. M.; Aly, A. S.; Gad, F. A.; Hassan, N. A.; El-Gazzar, A. B. A. Phosphorus, Sulfur and Silicon 2000, in press.

14. Khalil, Z. H.; Geies, A. A. Phosphorus, Sulfur and Silicon 1991, 60, 223.

Sample Availability: Available from the authors (or from MDPI).

(C) 2000 by MDPI (http://www.mdpi.org). Reproduction is permitted for noncommercial purposes. 